\title{
Nanostructures: Enhancing Potential Applications in Biomedicals
}

\author{
Shalini Singh \\ AMITY Institute of Biotechnology, AMITY University Uttar Pradesh, Lucknow, India. \\ Email: er.shalinisingh@gmail.com
}

Received September $9^{\text {th }}$, 2012; revised October $13^{\text {th }}, 2012$; accepted November $20^{\text {th }}, 2012$

\begin{abstract}
Nanotechnology is defined as the study and application of 1 - $100 \mathrm{~nm}$ sized structures. Nanomaterials have opened avenues for the industries and scientific endeavors. These recognized for unique size, dependant physical and chemical properties (optical, magnetic, catalytic, thermodynamic, electrochemical) [1]. Most significant properties of nanoparticles is their carbon strength. It is said to be so tough that recently with a nano-sized particles i.e. carbon nanotube-a bullet proof T-shirt/vests was manufactured. Nanotechnology was firstly proposed/initiated by Nobel Prize winner Richard Feynman in 1959 [2]. This science is credited to have applications ranging from electronics, biomedicals, food, fuel cells to biosensors and even fabrics. Though every field of science progressing but still faces some lacunae and that result in development of a new technology. The thriving biomedical techniques for disorders like cancers etc. is still in developmental stage where researchers and doctors are working hard for concrete therapeutic results from such nano-techniques. On cancers, the harmful side effects of its treatment like chemotherapy can't be left aside which is result of one of its drug delivery methods that don't pinpoint their intended target cells accurately rather affects whole area. Researchers in universities like Harvard and MIT have been able to attach special RNA strands, measuring about $10 \mathrm{~nm}$ in diameter, to nanoparticles and fill the nanoparticles with a chemotherapy drug. The RNA strands get attracted to cancer cells. When the nanoparticle encounters a cancer cell it adheres to it and releases the drug into the cancer cell. This directed method of drug delivery has great potential for treating cancer patients while producing less side harmful effects than those produced by conventional chemotherapy [3]. This paper provides valuable information to the researchers, knowledge experts and policy makers regarding the application of nanotechnology and its values in science and technology. Biomedical is one of the major issues which were catered by nanotechnology.
\end{abstract}

Keywords: Bionanotechnology; Nanoparticles; Medical Therapeutics; Cancers; Target Drug System; Nanoburrs; Quantum Dots

\section{Introduction}

Bionanotechnology is geared to create a huge impact in the benefit of society i.e. medical applications. It is actually being seen as a hope for future wellness and has offered better options in drug delivery system that makes the absorption of the components of medication more effective. Some of the medications are already using nanotechnology to effectively deliver their components to the areas that they need to get to include like controlling cholesterol levels, suppressing one's appetite for effective weight loss, etc.

Studies are onto the verge of developing customized nanoparticles that can deliver drugs directly to diseased cells in the body. After successful trials the method would surely reduce the damage occurred through treatment, what chemotherapy does to a patient's healthy cells [4].
In therapeutics, another area which is getting a lot of attention and where a lot of research is carried out, is the smart drugs. Smart drugs are intended to effectively target the areas and to eradicate completely the cause of the ailment occurring in a patient. There are a lot of ailments that will need this technique, however, the researchers are trying to come up with drug therapies that can help people get over illnesses faster simply because the drugs that they are taking work more effectively [5].

\section{Nanotechnology: Assisting Therapy and Methodology}

One of the ailments that are being seen to be benefited by miracles of nanotechnology is the Cancer (National Cancer Institute). NCI has created an alliance called the Alliance for Nanotechnology in Cancer, and the investments are made with a hope to find breakthrough tech- 
niques that can help in the early detection, diagnostics, and treatment of the different forms of such ailments.

Latest techniques and treatment methods for solid cancers have now become more tailored to the individual patient and to definite tumor type. Tumor markers are normally used to choose the suitability for specific medical therapies. Surgeries have become more tailored, more conservative in nature. Advances in imaging techniques allow accurate mapping of lesions improving preoperative planning and patient selection. Although, there are significant limitations to it as existing imaging modalities utilize non-specific contrast agents like Iodine-Based Contrast Agents used for Computed Tomography $(C T)$ causing significant toxicity. The use of radioisotopes for nuclear imaging has exposed the patients and healthcare workers to radiation, is stringently controlled by legislation. Nanotechnology may be the key to overcoming such limitations [6].

Nanoparticles that deliver chemotherapy drugs directly to cancer cells are under development. Tests are in progress for targeted delivery of chemotherapy drugs and their final approval for their use with cancer patients is pending. Cytimmune has come up with preliminary results of a Phase 1 Clinical Trial of their first targeted chemotherapy drug and another company, BIND Biosciences, has published the preliminary results of a Phase 1 Clinical Trial for their first targeted chemotherapy drug.

Thinking about imaging, Iron oxide nanoparticles can used to improve MRI images of cancer tumors. Intended nanoparticles coated with a peptide binds to a cancer tumor; once attached to the tumor the magnetic property of the iron oxide enhances the images from the Magnetic Resonance Imagining scan [7].

Another method developed to fight dermal cancer is where Gold Nanoparticles are utilized to which RNA molecules are attached. The nanoparticles are in an ointment that is applied to the skin. The nanoparticles penetrate the skin and the RNA attaches to a cancer related gene, stopping the gene from generating proteins that are used in the growth of skin cancer tumors.

A miraculous achievement where Nanoparticles get attached to the identified cancer cells in the blood stream may allow the cancer cells to be removed before they establish new tumors. In another study published in $\mathrm{Na}$ ture Medicine, a team led by Sam Gambhir, MD, Ph.D, professor and chair of radiology, showed that the minuscule nanoparticles engineered in his lab homed in on and highlighted brain tumors, precisely delineating their boundaries and greatly easing their complete removal. The new technique would help in improving the prognosis of patients with deadly brain cancers [8].

In a recent studies at the University School of Pharmacy, where protein-based polymers demonstrated a drastic potential for drug delivery and gene therapy in can- cers. Recombinant DNA technology that allows exploitation of polymers at molecular level revealed new possibilities for controlling the drug release process. The HPMA copolymers have demonstrated direct and effective delivery to Lewis Lung tumor cells in vitro and also in mice. These copolymers minimize uptake by non-target organs and overcome multi-drug resistance. Such polymers can also be correlated with gene release and transfer, for applications in cancer gene therapy. Further, a successful transport of PAMAM dendrimers across gastrointestinal epithelial cells with little or no toxicity is seen which directly indicates that polymer-based drugs can be delivered orally as well as intravenously. Such researches in nanoparticle polymer-based drug delivery will now focus on the influence of architecture, charge, and rug loading, and especially on animal testing for toxicity [9].

Nanoburrs are another therapeutic options designed to treat damaged arteries. Coated with tiny protein fragments that allows them to adhere to damaged artery walls and release drug (Paclitaxel) that inhibits cell division. Nanoburrs could also be used to treat arteriosclerosis and other cardiovascular issues and ailments [10].

A lot more areas are getting benefited through nanosciences and moreover it will be fruitful for those suffering from cardiovascular ailments, respiratory disorders, infertility, diabetes, brain disorders and even ageing in the future with the breakthroughs found in the research.

\section{Nanotechnology and Targeted Drug Release}

In therapeutics, a new category immerged, Self-assembling nanoscale vesicles that provide a stable, long-lasting encapsulation key to target drug delivery. The formed capsules attract, encapsulate and concentrate the charged organic drug molecules. This methodology is a key solution to the desired drugs that are not released until they reach their intended target. Such a nano-sized drug delivery system releases its payload in a specific $\mathrm{pH}$ condition, a feature that could prove particularly useful for targeted delivery of cancer treatments. It is known that the cancer drugs are highly toxic and are needed to be delivered specifically to the target i.e. tumour cells so as to avoid damage to the healthy tissues.

Targeted drug delivery systems using nanoparticles provide several advantages over conventional antibody guided therapy. Firstly, the drug load delivered to the target can be increased as several targeting ligands can be attached to a single particle and many drug molecules can be contained within the microstructure, known as nanovectors [11].

Nanotechnology initiated materials had overcome limitations that hinder other drug working on target-and- 
release technologies. The minute tube-shaped nanostructures possess the properties of ideal drug carriers: high drug presence, targets or probes, imaging capabilities, and non-toxicity. These magnetic structures prevent the need for bio-compatibility among components and enable the nanotubes to disperse easily in aqueous solutions. Such tiny capsules also protect their encapsulated medicine from attacks of enzymatic reactions.

There are several other examples in the literature of emerging nanosystems for drug and gene delivery where a multifunctional nanosystem. However, the magnetic nanoparticles can be used for magnetic resonance imaging $(M R I)$ where they could be used in vivo and in vitro approach to inhibit the tumor growth using doxorubicin linked to specific antibodies [12].

As suggested that the progress of such multifunctional particles could also be used to follow the target sites using MRI. Here the antibody moiety ensures specific targeting of cancer cells limiting any damage to the surrounding normal cells. Such nanosystems can also be deliberately utilized to introduce genes into cells [13].

Nanovectors as recent technologies have credit to carry multiple types of drugs, which would result in targeted combination therapy. Their ability to integrate allows access through biological barriers, leading to greater drug loads reaching the target cells. Liposomes, a nanovector use the leaky nature of tumor vasculature to concentrate at these sites. Liposome encapsulated doxorubicin has been used for the treatment of Kaposi's sarcoma, breast and ovarian cancers [14].

Nanofactories are another mechanized, nano-based drug synthesis and delivery system with MEMS devices that automatically collect raw materials in the local environment and synthesize drugs and deliver them to desired sites at specific times and dosages. It produces and delivers signal molecules that manipulate bacterial cells' "Quorum-Sensing”. As soon as the cells falsely perceive that there are sufficient numbers to become aggressive, they prematurely expose themselves to drug targeting or an immune response. Rather transporting an antibiotic, the nanofactories target athogenicity thus minimizing the pressure for bacteria to develop resistance. Besides advantageous nanofactory applications that reduces the costly animal testing, "Bio-Fabrication" putting synthetic pathways onto nanoparticles, which then mimic the reactions of biological systems. With the help of these manufactured fusion proteins covalently coupled and electro-deposited onto devices, the best applications of this finding would be applied in research of medical sciences and studies [15].

A collaborative research (reported in 2009) between US researchers at Northwestern University, Illinois and University of California, Los Angeles (UCLA) led to the development of "mechanized nanoparticles" that release their payload in response to a change in $\mathrm{pH}$. Under such provision, at heart, a mesoporous silica nanoparticle of around $200 \mathrm{~nm}$ in size that is capable of carrying drug and is easily absorbed by the cells. The required payload would leak through the particle's pores. These nanovalves are "stalks" that carry a ring, like a cyclodextrin or cucurbituril, and that controls the release of the cargo from the core of the mesoporous particle [16].

\section{Nanotechnology and Diagnostics}

Nanotechnology is contributing to the healthcare segment not only through novel therapy structures but also assisting the researchers in development of economical diagnostics procedures.

In diagnostics, apart from cancers treatments, nanoparticles are also exploited for multifunctional reasons. The large surface area of nanoparticles makes them efficient to accommodate several different functional groups on its surface. Conjugating different functional groups, such diagnostic (magnetic) and therapeutic (chemo) agents on to the same particle it would be possible to create dual or multifunctional nanoparticles, capable of diagnostic and therapeutic purpose simultaneously [17].

Attempts in diagnostics have also been made with a goal to combine imaging with therapy. In this direction, Radioimmunoconjugates were considered, but they were recognized to have lower tumor uptake, dose-limiting toxicity and exposed patients and medical personnel to ionizing radiation. A new interesting area of cancer nanotechnology has emerged out which encompasses cutting edge techniques from biology, chemistry, engineering and medicine. It is likely to evolve into a novel clinical diagnostic and therapeutic field, of clinical nanotechnology [18].

Also discussed above, Contrast agents like Iron Oxide; enhances MR images by altering the relaxation times of tissues in which the agent is present. They can also be used to label targeted molecular imaging probes. Unfortunately, no molecular imaging probe is currently available on the clinical MRI market. A promising platform for MRI contrast agent development is nanotechnology, where superparamagnetic iron oxide nanoparticles (SPIONS) are tailored for MR contrast enhancement, and/or for molecular imaging. SPIONs can be produced using a range of methods and the choice of method will be influenced by the characteristics most important for a particular application. In addition, the ability to attach molecular markers to SPIONS heralds their application in molecular imaging [19].

Nanosciences as medicine is also used for cell and bone regeneration. The research on prospects in cell and nerve regeneration is still in nascent stages, though nano sciences will prove it in reality and then can be used to 
help people who need to have certain cells in their body mended or bones that need fixing repaired.

Studies at the University of Illinois have shown that a nanoparticle combining aptamers (short strands of DNA or RNA which bind easily to biomolecules) and liposomes (drug-delivery vesicles) containing the drug Cisplatin for killing tumor cells. The aptamers binds to the nucleolin receptors found in certain breast cancer cells. A similar process could be used for Rheumatoid Arthritis [20,21].

Nanoparticles like CNT (carbon nanotubes) are used to increase the mechanical and electrical conductive properties of the tissue. They also improve the adhesive properties and control the release of GF (growth factors), and also give a physical shape to the tissue to create the desired structure [22].

Quantum Dots (crystalline nanoparticles) have been identified as means to identify the location of cancer cells in the body. It is advantageous in absolute quantitative profiling, spatial mapping, and multiplexing of cancer biomarkers. Studies have confirmed that after saturating all biomarkers on the cell surface, and, in conjunction with control experiments, they had extracted absolute quantitative values for the biomarker density in terms of the number of molecules per square micron on the cell surface. It is clear that quantitative spatial information of biomarker distribution on a single cell can be quantified, since the tumors' cell populations are inherently heterogeneous. We validate our quantitative measurements (number of molecules per square micron) using flow cytometry and demonstrate multiplexed quantitative profiling using color-coded QDs.

Quantitative targeting of pancreatic cancer biomarkers is demonstrated using quantum dot-antibody conjugates. Profiling and spatial mapping at the single cell level can be are achieved through minimizing non-specific binding and aggregation [23].

Another technique so called Scanning Near-Field Optical Microscopy (SNOM) has been utilized in conjunction with Quantum Dot labelling to interrogate the biomolecular composition of cell membranes. This technique overcomes the limits of optical diffraction found in standard fluorescence microscopy and also yields vital topographic information [24].

While many approaches to develop drug delivery vehicles have met with considerable success, the ability to fine tune them to only deliver drugs in response to specific biological conditions has been harder to attain.

\section{Nanotechnology and Pathogen Detectors}

Pathogen detection system (DeShong's) for Neisseria gonorrhoeae has reduced recognition time to minutes, when current methods require days. These nanoparticles coated with specific ligands can carry anti-tumor com- pounds directly to the desired site with no overt signs of toxicity. Such nano-scale materials sized to prevent removal by either the kidney or liver, used surface charges to optimize biological effectiveness, and attached receptors onto optical fibers to create novel biosensors. Now the team is working to develop this research into practical applications by optimizing materials, optimizing receptor quantities, vestigating new particle architectures, and determining toxicological effects [25,26].

Researchers at the University of Central Florida have devised a method where nanoparticles coated with DNA markers specific to the elusive pathogens. This system is effective and efficient than current methodology of picking up even small amounts of a pathogen. The most eciting feature of it is that it takes hours instead of weeks or months to deliver results [27].

\section{Conclusions}

The arena of Nanotechnology is new to this world and that too is still in its growth phase. Nanotechnology applications gather different fields such as environmental management, water resource, reduction in energy consumption, semi conductor devices, chemical catalysts and Nanorobots. But it had laid a great impact in integration with biology leading to the development of diagnostic devices, contrast agents, analytical tools, physical therapy applications, and drug delivery vehicles. Its role in bioimaging techniques and therapeutics stands remarkable. However, we are still demanding for more specific and accurate measures in bionanotechnology.

Medical technologies are focused on the developing micro and nano scale outputs. Concepts like lab-on-achip (microarrays techniques) are now exploited over the world over to facilitate ultra-sophisticated tests while with almost negligible amount of biological material from the patient. Nanoscale structures viz. Quantum Dots and Dendrimers have started with application in treating cancer where the silver nanoparticle is considered as major candidate against AIDS.

But a major concern here arises for the safety assessment of nanomaterials towards potential adverse health risks associated with nanotechnology products is needed. These structures may interact with living systems in ways that may be influenced by the nanoscale characteristics. Therefore to make these nanoscale products more safe and they do not pose any additional human health and environmental risks a risk assessment is required.

\section{REFERENCES}

[1] N. Sanvicens and M. P. Marco, "Multifunctional Nanoparticles-Properties and Prospects for Their Use in $\mathrm{Hu}-$ man Medicine," Trends in Biotechnology, Vol. 26, No. 8, 2008, pp. 425-433. doi:10.1016/j.tibtech.2008.04.005 
[2] R. P. Feynman, "There's Plenty of Room at the Bottom," Annual Meeting of the American Physical Society, Pasadena, 29 December 1959.

http://www.zyvex.com/nanotech/feynman.html

[3] http://expandthehorizon.wordpress.com/2012/08/09/nanot echnology-the-technology-at-atomic-scale/

[4] http://nanogloss.com/nanotechnology/nanotechnology-inmedicine/\#ixzz24GFyn4FQ

[5] R. Lobenberg, "Smart Materials, Applications of Nanotechnologyin Drug Delivery and Drug Targeting,” Proceedings of the International Conference on MEMS, NANO, and Smart Systems (ICMENS’03), Bannf, 20-23 July 2003.

[6] U. A. Gunasekera, Q. A. Pankhurst and M. Douek, "Imaging Applications of Nanotechnology in Cancer," Targeted Oncology, Vol. 4, No. 3, 2009, pp. 169-181. doi:10.1007/s11523-009-0118-9

[7] X.-H. Peng, et. al., "Targeted Magnetic Iron Oxide Nanoparticles for Tumor Imaging and Therapy,” International Journal of Nanomedicine, Vol. 3, No. 3, 2008, pp. 311321.

[8] B. Goldman, "Scientists Create Nanoparticles That Home in on Brain Tumor, Increasing Accuracy of Surgical Removal," 2012. http://med.stanford.edu/ism/2012/april/nanoparticle.html

[9] J. Gustafson and H. Ghandehari, "Silk-Elastinlike Protein Polymers for Matrix-Mediated Cancer Gene Therapy," Advanced Drug Delivery Reviews, Vol. 62, No. 15, 2010, pp. 1509-1523. doi:10.1016/j.addr.2010.04.006

[10] J. Rajiv, et al., "Nanoburrs: A Novel Approach in the Treatment of Cardiovascular Disease," International Research Journal of Pharmacy, Vol. 2, No. 5, 2011, pp. 9192.

[11] J. R. McCarthy and R. Weissleder, "Multifunctional Magnetic Nanoparticles for Targeted Imaging and Therapy," Advanced Drug Delivery Reviews, Vol. 60, No. 11, 2008, pp. 1241-1251. doi:10.1016/j.addr.2008.03.014

[12] J. Yang, C. H. Lee, H. J. Ko, J. S. Suh, H. G. Yoon, K. Lee, et al., "Multifunctional Magneto-Polymeric Nanohybrids for Targeted Detection and Synergistic Therapeutic Effects on Breast Cancer," Angewandte Chemie International Edition, Vol. 46, No. 46, 2007, pp. 8836-8839. doi:10.1002/anie.200703554

[13] Z. Medarova, W. Pham, C. Farrar, V. Petkova and A. Moore, "In Vivo Imaging of siRNA Delivery and Silencing in Tumors,” Nature Medicine, Vol. 13, No. 3, 2007, pp. 372-377. doi:10.1038/nm1486

[14] M. Ferrari, "Cancer Nanotechnology: Opportunities and Challenges,” Nature Reviews Cancer, Vol. 5, No. 3, 2005, pp. 161-171. doi:10.1038/nrc1566

[15] W. Bentley, "Nanofactories Enabling Nano-Based Drug Synthesis and Delivery." http://www.umbi.umd.edu/ cbr/lab_web/home.htm
[16] S. Angelos et al., "pH Clock-Operated Mechanized Nanoparticles," Journal of the American Chemical Society, Vol. 131, No. 36, 2009, pp. 12912-12914. doi:10.1021/ja9010157

[17] M. V. Yezhelyev, X. Gao, Y. Xing, A. Al-Hajj, S. Nie and R. M. O'Regan, "Emerging Use of Nanoparticles in Diagnosis and Treatment of Breast Cancer," Lancet Oncology, Vol. 7, No. 8, 2006, pp. 657-667. doi:10.1016/S1470-2045(06)70793-8

[18] C. Loo, A. Lowery, N. Halas, J. West and R. Drezek, "Immunotargeted Nanoshells for Integrated Cancer Imaging and Therapy,” Nano Letters, Vol. 5, No. 4, 2005, pp. 709-711. doi:10.1021/nl050127s

[19] J. Lodhia, et al., "Development and Use of Nanoparticles (Part-1): Synthesis of Nanoparticles for MRI," Biomedical Imaging and Intervention Journal, Vol. 6, No. 2, 2010, p. e12.

[20] Z. H. Cao, R. Tong, A. Mishra, W. C. Xu, G. C. L. Wong, J. J. Cheng and Y. Lu, "Reversible Cell-Specific Drug Delivery with Aptamer-Functionalized Liposomes,” Angewandte Chemie International Edition, Vol. 48, No. 35, 2009, pp. 6494-6498.

[21] L. J. Zhang and T. J. Webster, "Nanotechnology and Nanomaterials: Promises for Improved Tissue Regeneration,” Nano Today, Vol. 4, No. 1, 2009, pp. 66-68. doi:10.1016/j.nantod.2008.10.014

[22] A. Khushu, 2011. http://www.medlink-uk.org/Site/documents/Nano2011/K hushuA.pdf

[23] K. H. Lee, et al., "Quantitative Characterization of the Lipid Encapsulation of Quantum Dots for Biomedical Applications," Nanomedicine: Nanotechnology, Biology and Medicine, Vol. 8, No. 7, 2012, pp. 1190-1199.

[24] K.-A. D. Walker, C. Morgan, S. H. Doak and P. R. Dunstan, "Quantum Dots for Multiplexed Detection and Characterisation of Prostate Cancer Cells Using a Scanning Near-Field Optical Microscope,” PLoS ONE, Vol. 7, No. 2, 2012, e31592. doi:10.1371/journal.pone.0031592

[25] C. Fournier-Wirth and J. Coste, "Nanotechnologies for Pathogen Detection: Future Alternatives?” Biologicals, Vol. 38, No. 1, 2010, pp. 9-13. doi:10.1016/j.biologicals.2009.10.010

[26] P. DeShong, "Cell Surface Receptors Enabling Targeted Drug Delivery and Pathogen Detection.” http://www.umresearch.umd.edu/VPRPubfiles/Nanotechn ology\%20Medical\%20Applications.pdf

[27] C. Kaittanis, H. Boukhriss, S. Santra, S. A. Naser and J. M. Perez, "Rapid and Sensitive Detection of an Intracellular Pathogen in Human Peripheral Leukocytes with Hybridizing Magnetic Relaxation Nanosensors,” PLOS ONE, Vol. 7, No. 4, 2012, e35326. doi:10.1371/journal.pone.0035326 